Coulomb charging effects in an open quantum dot device

This article has been downloaded from IOPscience. Please scroll down to see the full text article. 2001 J. Phys.: Condens. Matter 139515

(http://iopscience.iop.org/0953-8984/13/42/312)

The Table of Contents and more related content is available

Download details:

IP Address: 140.112.113.225

The article was downloaded on 21/07/2009 at 07:16

Please note that terms and conditions apply. 


\title{
Coulomb charging effects in an open quantum dot device
}

\author{
O A Tkachenko ${ }^{1,2}$, V A Tkachenko ${ }^{1}$, D G Baksheyev ${ }^{1}$, C-T Liang ${ }^{3}$, \\ M Y Simmons ${ }^{4}$, C G Smith ${ }^{5}$, D A Ritchie ${ }^{5}$, Gil-Ho Kim ${ }^{6}$ and M Pepper \\ ${ }^{1}$ Institute of Semiconductor Physics, Novosibirsk, 630090, Russia \\ ${ }^{2}$ Novosibirsk State University, Novosibirsk, 630090, Russia \\ ${ }^{3}$ Department of Physics, National Taiwan University, Taipei 106, Taiwan \\ ${ }^{4}$ School of Physics, University of New South Wales, Sydney 2052, Australia \\ 5 Cavendish Laboratory, Madingley Road, Cambridge CB3 OHE, UK \\ 6 Telecommunication Basic Research Laboratory, ETRI, PO Box 106, Daejon 305-600, Korea \\ E-mail: ctliang@phys.ntu.edu.tw (C-T Liang)
}

Received 23 August 2001

Published 5 October 2001

Online at stacks.iop.org/JPhysCM/13/9515

\begin{abstract}
In this work we clarify the nature of frequent oscillations of the conductance of open quantum dots that were reported by Liang et al (Liang C-T et al 1998 Phys. Rev. Lett. 81 3507). Continuous and almost periodic oscillations superimposed upon ballistic conductance features are observed when the conductance $G$ of the dot changes within a wide range $0<G<6 e^{2} / h$. We confirm the single-electron origin of the conductance oscillations by means of measurements in a perpendicular magnetic field and calculation of capacitances of the quantum dot with respect to two-dimensional (2D) electron gas reservoirs and gates. The calculations of the three-dimensional electrostatics of the device and $2 \mathrm{D}$ transport through the dot show that the progression of the Coulomb oscillations into the region $G>2 e^{2} / h$ is the consequence of suppression of inter-one-dimensional-subband scattering. The theory of Coulomb blockade and the Landauer formula are modified for the case of the quasi-one-dimensional system to describe combined charging and ballistic transport through the dot. Measured dependences of the conductance on the gate voltages and its temperature behaviour are correctly reproduced by the calculations.
\end{abstract}

\section{Introduction}

Charge quantization plays a central role in electron transport through lateral quantum dots weakly coupled to leads. It had been commonly believed, and argued for on the basis of the validity of Coulomb blockade theory [1,2] and various experimental results [3-13], 
that Coulomb blockade oscillations of the conductance weaken and gradually vanish as the transparency of the barriers increases up to the conductance quantum $2 e^{2} / h$. However, the possibility of Coulomb charging in open quantum dots is now being intensively investigated both theoretically [14-17] and experimentally [18-23]. In a series of papers, single-electron charging was studied for the dot with one fully transmitting point-contact lead, the other contact having the resistance $R>h / e^{2}[16,21,22]$. However, of special interest are charging effects when the total conductance of the device is greater than $2 e^{2} / h[19,20,23]$. In strong magnetic fields, continuous Coulomb oscillations superimposed upon large-period oscillations have been detected with the conductance $G$ ranging up to $G=3 e^{2} / h$ [19]. Only recently has similar experimental evidence of single-electron charging of an open quantum dot been obtained in zero magnetic field [23]. The quantum dot was defined by two side gates, which deplete electrons within the channel, and three narrow overlying finger gates. The outermost finger gates introduce the entrance and exit barriers to the dot, and the central finger gate stabilizes the depth of the potential inside the dot. The impurity scattering in the device, fabricated on an ultrahigh-quality high-electron-mobility transistor (HEMT), is negligible. Surprisingly, continuous and periodic oscillations superimposed upon ballistic conductance steps were observed when the conductance through the dot changed within a wide range $0<G<6 e^{2} / h$. A smooth transition of the oscillations from $G>2 e^{2} / h$ to $G<2 e^{2} / h$ with decreasing barrier transparency leads to the conclusion that the oscillations are due to singleelectron charging of the quantum dot [23]. However, none of the existing theories can explain the manifestation of single-electron oscillations over such a wide range of the conductance.

In this paper we provide additional experimental evidence confirming the charge nature of the observed effect and explain, on the basis of realistic modelling, why the oscillations penetrate far into the region $G>2 e^{2} / h$. In the experimental part of the paper we note that the observed frequent oscillations look similar near the onset of conductance and in the open dot regime - that is, the oscillations remain smoothed and their period fluctuates within the whole range $0<G<6 e^{2} / h$ in contrast to Coulomb blockade peaks, sharp and equally spaced, observed in other quantum dots $[4-13,18,22]$. This behaviour could not be explained by orthodox theory $[1,2]$, so we provide an additional confirmation of the Coulomb nature of the oscillations by observing that the phase of the oscillations at $G<2 e^{2} / h$ does not shift in a perpendicular magnetic field. We show that the phase of the oscillations versus sidegate voltage uniformly shifts with increasing finger-gate voltages, as it should for charging oscillations. We also report similar frequent conductance oscillations observed as a function of central finger-gate voltage and superimposed on the background of regular large-scale peaks.

In the theoretical part of the paper we report realistic modelling of the $3 \mathrm{D}$ electrostatics and electron transport in the quantum dot. By calculating the capacitances of the quantum dot with respect to the gates and two-dimensional electron gas (2DEG) reservoirs, we confirm the single-electron origin of the frequent conductance oscillations. The modelling of the 2D electron transport shows that these oscillations are not due to resonant transmission because in experiment they are far more frequent than the resonant peaks in the calculations. Instead, the resonances of double-barrier Fabry-Pérot interference are associated with the large-scale resonant features in the background conductance. Thus, in this device single-electron charging and coherent electron transmission at $0<G<6 e^{2} / h$ coexist. In the following we discuss how, under special circumstances, Coulomb charging effects can be present in open dots. Our calculations demonstrate the unique versatility of this dot geometry with adjustable voltages on the side gates and three finger gates. We show that in some voltage regimes the electrostatic potential in the plane of the 2DEG is separable as $U(x, y)=U_{1}(x)+U_{2}(y)$ and thus the device exhibits simple one-dimensional behaviour. In standard quantum dots where the constrictions are defined by two pairs of split gates, Coulomb oscillations in zero magnetic 
field are only observable at $G<2 e^{2} / h$. In order to determine the difference between this new device and the standard quantum dot, we compare the calculated electrostatics and transport in the two different types of dot. Our results show that inter-1D-subband scattering is suppressed in the new type of open quantum dot, owing to the special design, whereas in the standard quantum dots the inter-subband mixing is considerably enhanced once the transmission route via the first subband is opened. We argue that in a $1 \mathrm{D}$ system, sequential tunnelling via weakly transmitted subbands and high sensitivity of the barrier transparency in the constrictions to the variations of the Fermi level in the dot make it possible to observe the effects of Coulomb charging at $G>2 e^{2} / h$. We modify the theory of Coulomb blockade and the Landauer formula to model single-electron oscillations and the temperature dependence of all the features of the conductance.

This paper is organized as follows. In section 2 the quantum dot device is described, and the conductance of the dot versus central finger-gate voltage and the influence of magnetic field on the observed oscillations are reported. Modelling of the basic properties of the structure is described in section 3. First we discuss the electrostatics of the device (sections 3.1 and 3.2). Then the calculated two-dimensional potential profile is used for modelling multiple-mode electron transmission through the quantum dot (section 3.3). In section 3.4 we describe the modification of Coulomb blockade theory used to simulate the observed conductance curves. In section 3.5 we show that the observed oscillations are determined not by the current through the localized states, but by charge-induced modulation of the ballistic current.

\section{Experiment}

The two-layered finger-gate pattern shown in the inset to figure 1 was defined by electron beam lithography on the surface of a high-mobility $\mathrm{GaAs} / \mathrm{Al}_{0.33} \mathrm{Ga}_{0.67}$ As heterostructure, $157 \mathrm{~nm}$ above a 2DEG. There is a $30 \mathrm{~nm}$ thick layer of polymethylmethacrylate (PMMA) which has been highly dosed by an electron beam, to act as a dielectric [24] between the Schottky split gate (SG) and three finger gates (F1, F2 and F3) so that all gates can be independently controlled.

After brief illumination by a red light-emitting diode, the carrier concentration of the 2DEG was $1.6 \times 10^{15} \mathrm{~m}^{-2}$ with a mobility of $250 \mathrm{~m}^{2} \mathrm{~V}^{-1} \mathrm{~s}^{-1}$. The corresponding transport mean free path is $16.5 \mu \mathrm{m}$, much longer than the effective 1D channel length. Experiments were performed in a dilution refrigerator at $T=50 \mathrm{mK}$ and the two-terminal conductance $G=\mathrm{d} I / \mathrm{d} V$ was measured using an ac excitation voltage of $10 \mu \mathrm{V}$ with standard phasesensitive techniques. In all cases, a zero-split-gate-voltage series resistance $(\approx 900 \Omega)$ was subtracted. Two samples, for five different coolings, show similar characteristics.

When a constriction was formed in the centre or at any end of the channel by changing the voltage on an appropriate gate (SG or F2, F1 or F3), distinct quantization of the ballistic conductance was observed (figure 1 in reference [23]). Absence of resonant features in the transition regions and plateaux of the conductance indicate that a clean 1D channel is obtained in which impurity scattering is negligible [23]. The quantum dot was formed only when large negative voltages applied to both $\mathrm{F} 1$ and $\mathrm{F} 3\left(V_{\mathrm{F} 1} \approx V_{\mathrm{F} 3} \approx-2 \mathrm{~V}\right)$ introduced the entrance and exit barriers to the dot. In that case, split-gate dependences of the conductance at $T=50 \mathrm{mK}$ displayed resonant features: frequent quasiperiodic oscillations superimposed upon the background of wide steps and peaks in the conductance range $0<G<6 e^{2} / h$ (figures 2 and 3 in reference [23]). The oscillations disappeared at the critical temperatures, which were decreasing as the value of the background conductance increased (cf. figure 2 in reference [23]): $2 \mathrm{~K}$ at $G<e^{2} / h, 1 \mathrm{~K}$ at $e^{2} / h<G<2 e^{2} / h, 0.3 \mathrm{~K}$ at $2 e^{2} / h<G<4 e^{2} / h$ and $0.15 \mathrm{~K}$ at $4 e^{2} / h<G<6 e^{2} / h$. 


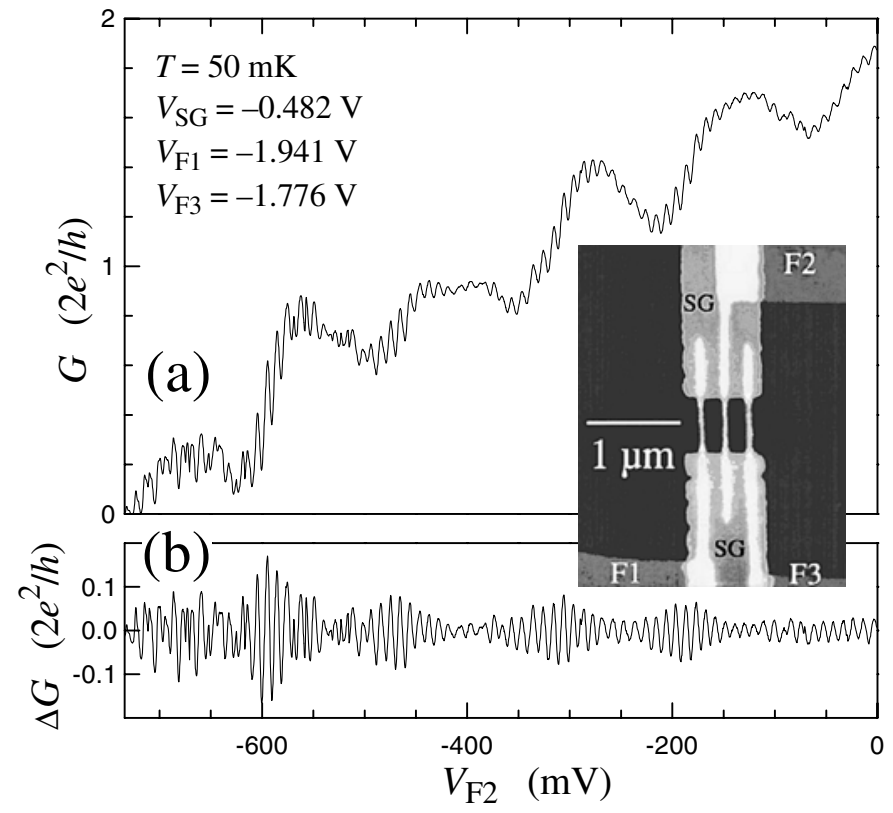

Figure 1. (a) $G\left(V_{\mathrm{F} 2}\right)$ for fixed outermost finger-gate voltages $V_{\mathrm{F} 1,3}$ and split-gate voltage $V_{\mathrm{SG}}$. (b) The oscillations without the background of large-scale ballistic conductance features, as extracted from (a), showing the modulation of the amplitude and unstable periodicity. The inset shows a scanning electron micrograph of a typical device. The brightest regions correspond to finger gates with joining pads, labelled as F1, F2 and F3, lying above the split gate (labelled as $\mathrm{SG})$, with an insulating layer of cross-linked PMMA in between.

Here we report typical traces of the full conductance $G$ measured as a function of the central finger-gate voltage $V_{\mathrm{F} 2}$, with the split-gate voltage $V_{\mathrm{SG}}$ fixed (figure 1(a)). The frequent oscillations are similar to those previously reported [23]. The peaks at $G<e^{2} / h$ $\left(V_{\mathrm{F} 2}<-0.5 \mathrm{~V}\right)$ are not as sharp and equally spaced as one would expect from the theory of Coulomb blockade. Furthermore, the observed oscillations overlie the wide maxima of the background conductance which appear periodically with $V_{\mathrm{F} 2}$. The large-scale quasiperiodic conductance peaks covered by equally spaced peaks of the Coulomb blockade at $G<e^{2} / h$ have already been reported [12] and interpreted as Fabry-Pérot resonances due to coherent electron tunnelling through the quantum dot. In our case the frequent small-amplitude oscillations demonstrate abrupt phase changes at $G<e^{2} / h$ (figure 1(b)) and penetrate into the region $G>2 e^{2} / h$, where the transport is traditionally considered coherent and Coulomb charging effects are not usually observed. Notice also that the amplitude of frequent oscillations increases on the slopes of background conductance. This modulation of amplitude will be explained in section 3.5 .

To check that the frequent oscillations are connected to charge quantization, we studied the conductance of the dot in a perpendicular magnetic field. Figure 2 shows dependences $G\left(V_{\mathrm{SG}}\right)$ measured at fixed finger-gate voltages and $T=50 \mathrm{mK}$ as the magnetic field changed from $B=0$ to $B=20 \mathrm{mT}$ for the main set-up (a) and to $B=160 \mathrm{mT}$ for set-up (b). In the case shown in figure 2(a) the oscillations do not shift along $V_{\mathrm{SG}}$ when the magnetic flux penetrating the dot area changes within 1.5 flux quanta. The oscillations at $G>2 e^{2} / h$ are suppressed with increasing $B$. Figure 2(b) illustrates the generic case where the oscillations at $G<2 e^{2} / h$ retained their position in a wide range of $B$ ( $>10$ flux quanta). 

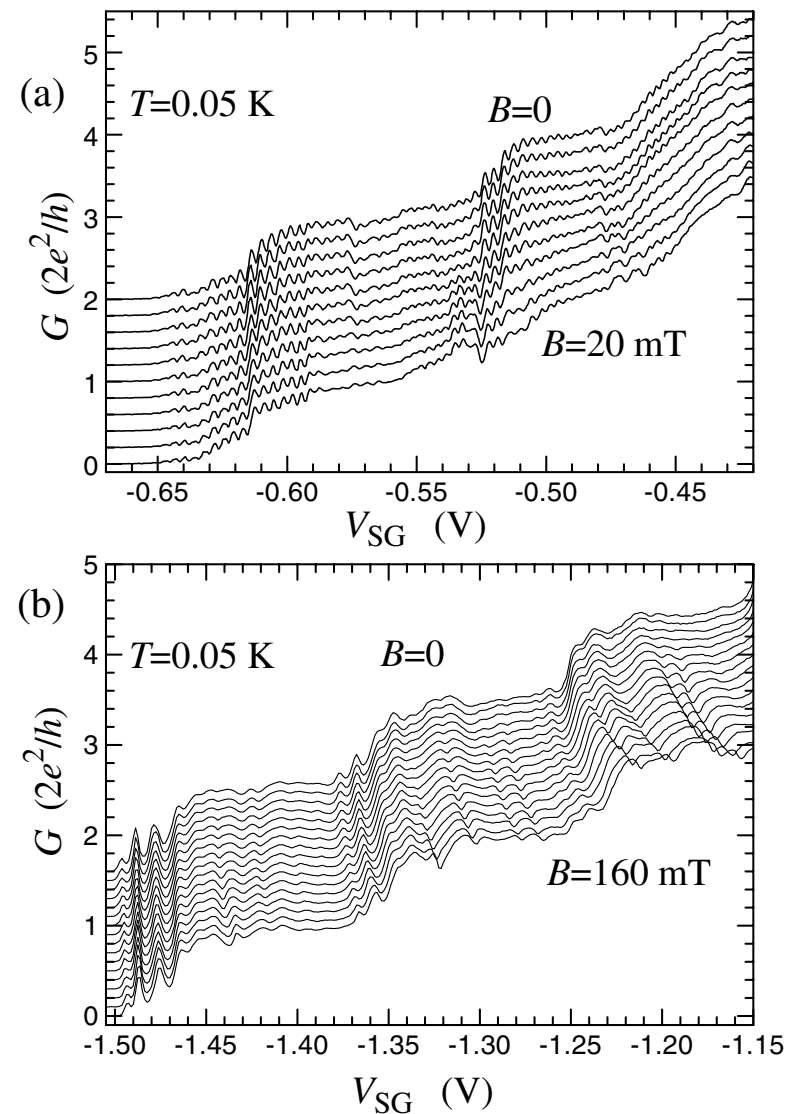

Figure 2. $G\left(V_{\mathrm{SG}}\right)$ for fixed finger-gate voltages with a perpendicular magnetic field changing from $B=0$ to $B=20 \mathrm{mT}$ for set-up (a) and to $B=160 \mathrm{mT}$ for set-up (b). The curves are vertically offset for clarity.

It is known that the shift of the Coulomb peaks is controlled by the polarization charge $q_{0}$ which is usually assumed constant in the theory of Coulomb blockade [1]. Theory [2] and earlier experiments with isolated quantum dots have shown that the position of Coulomb oscillations is insensitive to perpendicular magnetic field in a wide range. The oscillations at $G<2 e^{2} / h$ that we study in this paper demonstrate similar phase stability, indicating their underlying charge-quantization origin. In reference [23] the oscillations were shown to evolve gradually from region $G<2 e^{2} / h$ to $G>2 e^{2} / h$ with the period conserved (see below) as the barrier transparency was increased. Therefore the period of the oscillations for the open dot also corresponds to the change of the dot charge by one electron.

Further evidence of the electrostatic single-electron nature of the effect is derived by observing the evolution of conductance traces $G\left(V_{\mathrm{SG}}\right)$ with barrier transparency. Figure 3 demonstrates how (a) the background conductance $\bar{G}_{\mathrm{RA}}\left(V_{\mathrm{SG}}\right)$ and (b) the oscillations less the background change with incremental voltage steps on the outermost finger gates $V_{\mathrm{F} 1}$ and $V_{\mathrm{F} 3}$ at $T=50 \mathrm{mK}$. One can see that the oscillations of $G\left(V_{\mathrm{SG}}\right)$ gradually evolve with similar shape and periodicity from the region $G>2 e^{2} / h$ to the region $G<2 e^{2} / h$, indicating their common physical origin. Note that the modulation of the oscillation amplitude in figure 3(b) allows us to trace the movement of the oscillations along parallel lines. Thus we show that the phase 

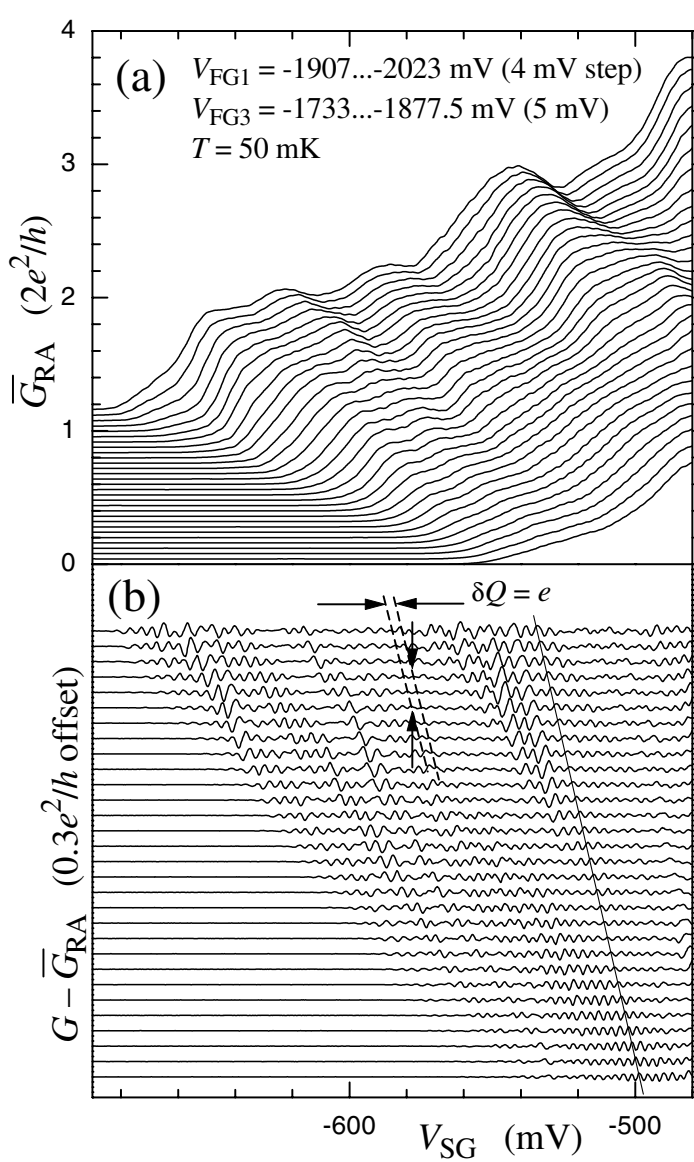

Figure 3. Evolution of conductance $G\left(V_{\mathrm{SG}}\right)$ with transparency of the barriers. (a) The running average of the conductance of the quantum dot over $7.8 \mathrm{mV}$ and (b) the oscillations with background subtracted. Curves are successively vertically displaced for clarity.

of the oscillations depends linearly on $V_{\mathrm{F}}$. This corresponds to the conservation of the dot charge $Q=n e$ as defined by the simple electrostatic relation $C_{\mathrm{SG}} V_{\mathrm{SG}}+C_{\mathrm{FG}} V_{\mathrm{F}}+q_{0}+n e=0$. Transition to a neighbouring parallel line implies change of $n$ by unity. Two such lines are shown dashed in figure 3(b), where the horizontal and vertical separations between them obey the same condition, $\delta Q=e$. The condition $\delta Q=e$ can be used to find the capacitances between the dot and the gates of the sample (table 1). The relation $\delta Q=e$ will be tested numerically in section 3.1, where we also calculate all the capacitances. Further analysis of the dependence of the oscillations and background in the $\left(V_{\mathrm{SG}}, V_{\mathrm{F}}\right)$ parameter plane allows us to extract from experimental data qualitative information about ballistic transport which will be compared with the modelling in sections 3.3 and 3.4.

Summarizing this section, the frequent oscillations observed in the conductance of the open dot are uniform over the whole measurement range. Experimental evidence of their single-electron nature is obtained. However, unusual behaviour of the oscillations, even in the vicinity of pinch-off, precludes us from ascribing them to Coulomb blockade of sequential tunnelling currents. The properties of the background conductance and oscillations will be the subject of the theoretical examination below. 
Table 1. Calculated and measured gate-voltage periods of single-electron oscillations. In the calculations, $V_{\mathrm{F} 1}$ and $V_{\mathrm{F} 3}$ were changed simultaneously, $V_{\mathrm{F} 1}=V_{\mathrm{F} 3}=V_{\mathrm{F}}$, while experimentally the two finger gates were controlled independently. Thus, $\Delta V_{\mathrm{F}}$ should be compared with $\frac{1}{2} \times \frac{1}{2}\left(\Delta V_{\mathrm{F} 1}+\Delta V_{\mathrm{F} 3}\right)$.

\begin{tabular}{lll}
\hline Period of oscillations & Calculated $(\mathrm{mV})$ & Measured $(\mathrm{mV})$ \\
\hline$\Delta V_{\mathrm{SG}}$ & $2.8-4.5$ & $3.6 \pm 1$ \\
$\Delta V_{\mathrm{F}}$ & $6.6-11.5$ & \\
$\Delta V_{\mathrm{F} 1}$ & & 23.8 \\
$\Delta V_{\mathrm{F} 3}$ & & 25.9 \\
$\Delta V_{\mathrm{F} 2}$ & $6.6-9$ & 8.7 \\
\hline
\end{tabular}

\section{Numerical results and discussion}

\subsection{Electrostatics}

In order to check the correspondence between the observed period of oscillations and the change of the dot charge by one electron, and to obtain an estimate of the charging energy, we calculated the capacitance of the dot with respect to the contacts, fingers and split gates. The electrostatic potential profile in the device was determined by solution of the 3D Poisson equation with a local 2DEG density given by the 2D Thomas-Fermi approximation assuming a boundary condition of frozen charge for the surface states and impurities. It was checked that fluctuation potential in this structure due to ionized dopants is absent due to the wide AlGaAs spacer $(100 \mathrm{~nm})$. The calculation technique has been described in references [25,26]. The modelling has been carried out for the main set-up, for which all the results presented in reference [23] and in figures 1, 2(a) and 3 were obtained.

The conformity of this fairly simple model to the experiment was checked by calculation of the pinch-off voltages. In the calculations, at $V_{\mathrm{F}}=0$ the channel pinches off when $V_{\mathrm{SG}}=-1.8 \mathrm{~V}$ (the same as in the experiment). When $V_{\mathrm{SG}}=-0.7 \mathrm{~V}$ the finger gates raise the potential barriers in the constrictions above the Fermi level at $V_{\mathrm{F}}=-1.4 \mathrm{~V}$ (experimentally the split gate pinches off at $V_{\mathrm{SG}}=-0.7 \mathrm{~V}$ when $V_{\mathrm{F} 1}=-1.9 \mathrm{~V}$ and $\left.V_{\mathrm{F} 3}=-1.7 \mathrm{~V}\right)$. We ascribe this small difference between the calculated $\left(V_{\mathrm{F}}\right)$ and experimental $\left(V_{\mathrm{F} 1}, V_{\mathrm{F} 3}\right)$ values to the fact that we do not take into account the capacitances of the finger gates with respect to the shield of the structure (we also neglect electric field lines going above the PMMA layer).

We calculated the potential profile, charge distribution and the total charge of the dot, as well as the capacitances in the ranges $V_{\mathrm{SG}}=-0.75$ to $-0.5 \mathrm{~V}$ and $V_{\mathrm{F}}=-1.3$ to $-1.4 \mathrm{~V}$. This parameter region agrees with the ranges of experimental gate voltages specified in figure 3 . Figure 4 shows the charge density for a quarter of the quantum dot for closed and open states. Transverse cross sections of the electrostatic potential in the 2DEG are shown in insets for two different coordinates along the channel: in the centre of the dot, and directly beneath the outermost gates. On changing the voltage $V_{\mathrm{SG}}$, the dot transforms from a closed state (a) to an open state (b), with a corresponding change in the width of both dot and constriction. Figure 5(a) shows that the voltage on the outermost finger gates controls both the height of the barriers and the width of the constrictions with little change in the depth of the dot. At large finger-gate voltages $V_{\mathrm{F} 1,3}=-1.3$ to $-1.4 \mathrm{~V}$ and low split-gate voltages $V_{\mathrm{SG}} \approx-0.5 \mathrm{~V}$, the transverse potential profile of the constriction resembles a rectangular well rather than a parabola. With the central finger gate kept at zero voltage, the width and depth of the quantum dot were found to depend on $V_{\mathrm{SG}}$ only. On the other hand, if the voltage on the outermost finger gates is fixed and the central finger-gate voltage is varied, it mainly changes the depth of the potential in the quantum dot (figures 5(b), 5(c)). 

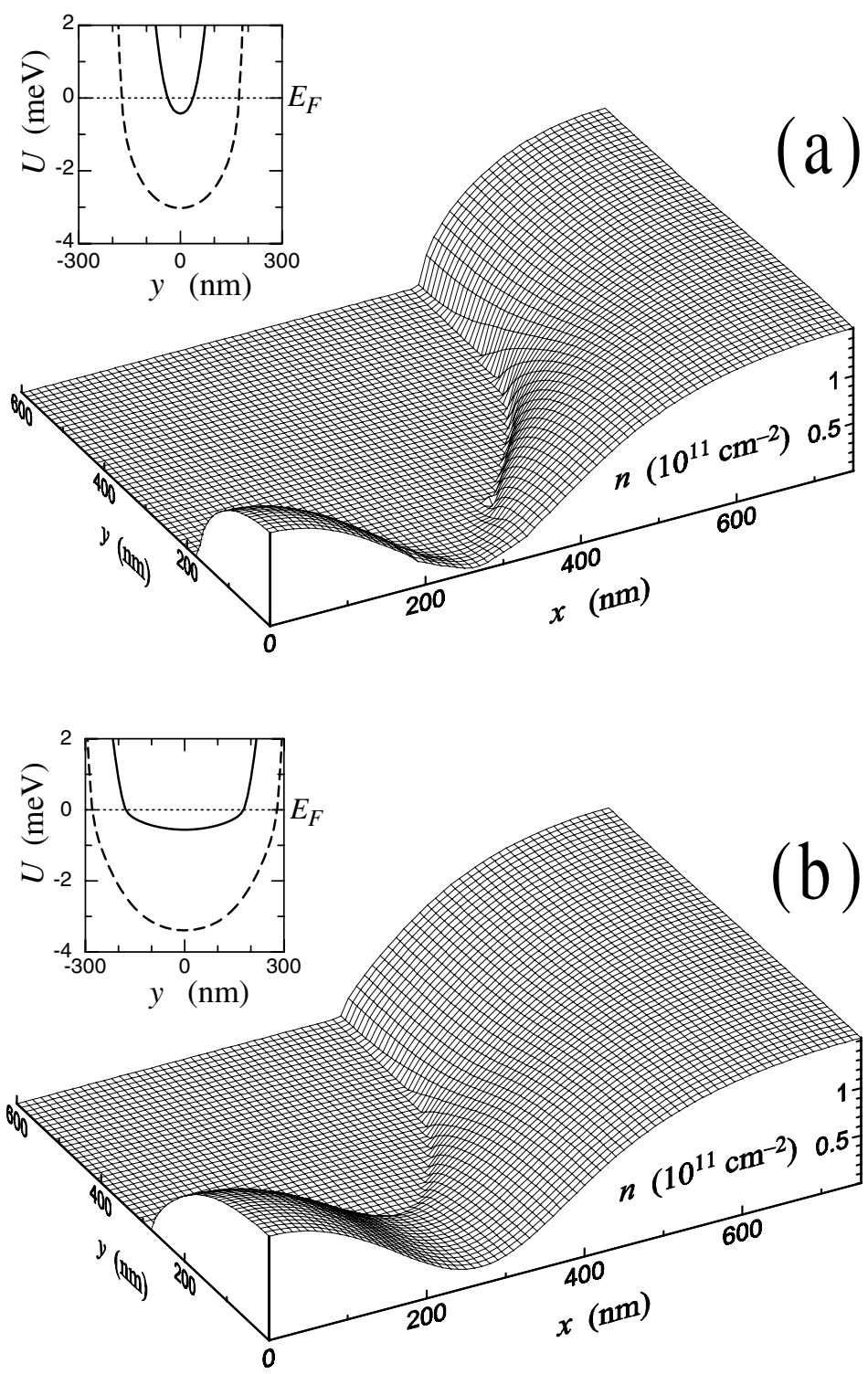

Figure 4. 3D plots of charge density in the quantum dot: (a) closed dot, $V_{\mathrm{SG}}=-0.75 \mathrm{~V}$, $V_{\mathrm{F}}=-1.3 \mathrm{~V}$; (b) open dot, $V_{\mathrm{SG}}=-0.5 \mathrm{~V}, V_{\mathrm{F}}=-1.3 \mathrm{~V}$. The insets show cross sections of the potential in the centre of the dot, at $x=0$ (dashed line), and in the constriction, at $x=270 \mathrm{~nm}$ (solid line).

The calculations show that the number of electrons in the dot changes from 80 to 140 as the split-gate voltage changes from $V_{\mathrm{SG}}=-0.7 \mathrm{~V}$ to $-0.5 \mathrm{~V}$ (with fixed $V_{\mathrm{F} 1}=V_{\mathrm{F} 3}=-1.3 \mathrm{~V}$ ). This change in the number of electrons corresponds to the number of oscillations observed on the upper curve in figure 3(b). Calculated capacitances of the dot to the gates are also close to the experimentally estimated ones and lie within the measured period variation (table 1). Thus, the conclusion that each oscillation of the conductance reflects the change of the dot charge by one electron is confirmed. 
(a)

(b)
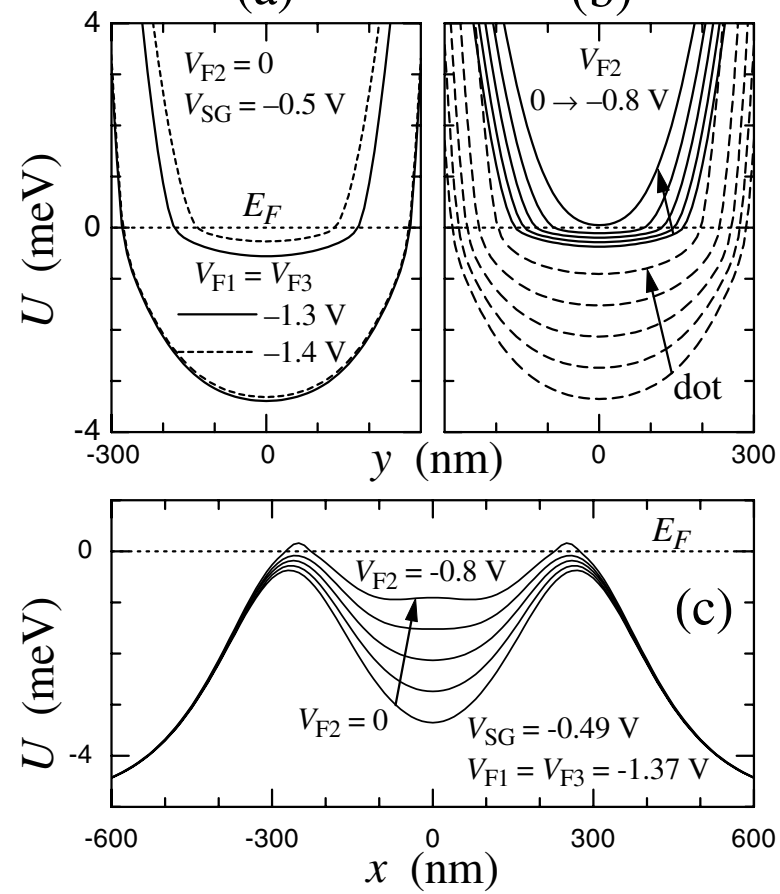

Figure 5. The effect of finger gates on the electrostatic potential in the dot with fixed split-gate voltage. (a) Transverse cross sections of the potential in the dot and in the constrictions for two cases, defined by gate voltages $V_{\mathrm{SG}}=-0.5 \mathrm{~V}, V_{\mathrm{F} 2}=0, V_{\mathrm{F}}=-1.3 \mathrm{~V}$ (solid lines) and $V_{\mathrm{F}}=-1.4 \mathrm{~V}$ (dashed lines). ((b), (c)) Deformation of the dot (defined by fixed voltages $V_{\mathrm{SG}}=-0.49 \mathrm{~V}$, $V_{\mathrm{F} 1,3}=-1.37 \mathrm{~V}$ ) with variation of $V_{\mathrm{F} 2}$ from $-0.8 \mathrm{~V}$ to zero with $0.2 \mathrm{~V}$ steps.

Introducing a small Fermi level difference between the dot and the 2DEG reservoirs, we calculated the capacitance of the dot with respect to both contacts as $C_{\mathrm{r}}=340-370 \mathrm{aF}$ for an almost closed quantum dot. The capacitance is doubled when three 1D subbands become transmitted. Thus, this capacitance is almost an order of magnitude higher than that to the gates and cannot be neglected, so the charging energy is $e^{2} / 2 C=0.1-0.2 \mathrm{meV}$, where $C=C_{\mathrm{r}}+e / \Delta V_{\mathrm{SG}}+e / \Delta V_{\mathrm{F}}+e / \Delta V_{\mathrm{F} 2}$, comparable to the thermal broadening at $T \approx 1-2 \mathrm{~K}$. In reference [23] the oscillations near the pinch-off are shown to persist up to $1 \mathrm{~K}$, in accordance with the conventional theory of Coulomb blockade. The decrease of the charging energy to $0.1 \mathrm{meV}$ at $G \sim 6 e^{2} / h$, as found in the calculations of the electrostatics, should lower the limiting temperature for observing the oscillations in this range to $\sim 0.5 \mathrm{~K}$. In reality the measured temperature is still three times smaller [23]. We will explain this strong reduction in section 3.5 .

\subsection{Comparing quantum dots of different types}

To understand the difference between the dot under study (figure 6(a)) and a standard quantum dot (where the constrictions are induced by two pairs of split gates and Coulomb oscillations are observed only at $\left.G<e^{2} / h\right)$, calculations of the electrostatics were also carried out for the case in which the outermost $160 \mathrm{~nm}$ wide finger gates were separated by a $260 \mathrm{~nm}$ gap (figure 6(b)). We will denote those devices as A and B, respectively. Except for the finger 

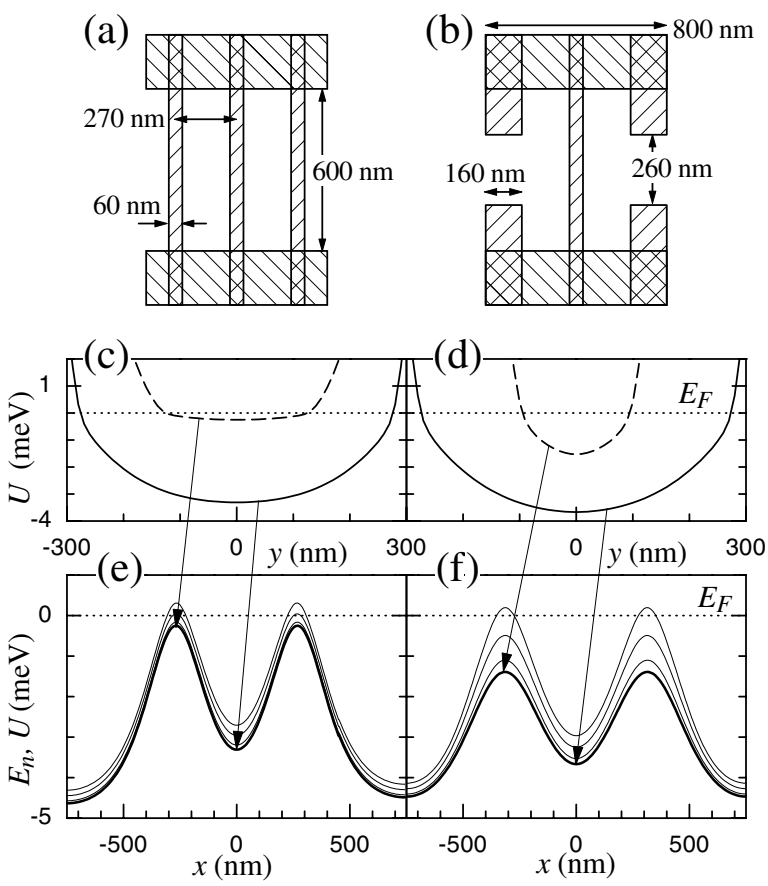

Figure 6. Comparison of potential profiles for two devices: ((a), (c), (e)) quantum dot with overlying finger gates, type A; ((b), (d), (f)) quantum dot with broken finger gates, type B. $((\mathrm{c}),(\mathrm{d}))$ The transverse cross section in the dot $(x=0$, solid lines $)$ and in the constrictions ( $x=270 \mathrm{~nm}$, dashed lines). ((e), (f)) The longitudinal potential $U(x, y=0)$ shown by a thick solid line and the three lowest $1 \mathrm{D}$ subbands $E_{n}(x)$.

gates, all the parameters of devices A and B are the same. The calculated capacitances of the quantum dot in closed and open states for cases A and B are similar. The essential differences between the electrostatic potentials in the plane of the 2DEG only appear in the constrictions. In device $\mathrm{B}$, the barriers in the constrictions $x=x_{\mathrm{c}}$ are lower, and the transverse cross section of potential there resembles a deep and narrow parabola $U\left(x_{\mathrm{c}}, y\right)=U_{\mathrm{c}}+\frac{1}{2} m \omega_{\mathrm{c}}^{2} y^{2}$ with energy quantum $\hbar \omega_{\mathrm{c}}=0.6-0.8 \mathrm{meV}$ (figures $6(\mathrm{~d}), 6(\mathrm{f})$ ). The quantum in the centre of the channel $x_{\mathrm{d}}=0$ (the quantum dot) is 2-3 times smaller. In device A the transverse potential in the constriction resembles a cut parabola (figure 6(c)), so the lowest 1D subbands are denser near the bottom, like that in a rectangular potential well. When the quantum dot is open for transmission via the first subband, the $1 \mathrm{D}$ subband spacing in device $\mathrm{A}$ is almost equal inside the dot and constrictions: $\hbar \omega_{\mathrm{c}} \approx \hbar \omega_{\mathrm{d}}=0.2-0.3 \mathrm{meV}$ (figure 6(e)).

The energy levels of transverse quantization $E_{n}(x)$ were determined from a solution of the Schrödinger equation for the calculated electrostatic potential $U(x, y)$ by a tight-binding method $[25,26]$. To impose zero boundary conditions for transverse motion, infinite walls were put at $600 \mathrm{~nm}$ from the axis of the channel. The picture of 1D subbands shows how the subband spacing changes along the channel axis and how many subbands are open for transmission through the quantum dot at a given Fermi level.

Figures 6(e), 6(f) show the positions of the three lowest 1D subbands $E_{n}(x)$ for devices A and B. The Fermi level is shown by a dotted line and corresponds to the zero energy. In case A, the subband spacing is almost independent of $x$. This means that the transverse cross sections of potential in the dot and in the constrictions have the same parabolic shape, in other 
words $U(x, y)=U(x)+\frac{1}{2} m \omega_{\mathrm{c}}^{2} y^{2}$. Then the variables $x$ and $y$ in the Schrödinger equation are separated, and the motion along $x$ - and $y$-directions is described by separate equations, with no mixing between different $1 \mathrm{D}$ subbands. Thus the transmission problem reduces to a one-dimensional one.

In contrast, in device $\mathrm{B}$, where there is a gap between the finger gates, 1D subbands are not equally spaced in energy and the inter-subband spacing changes by 2-3 times along the channel (figure 6(f)). Thus the potential has such a shape that the variables in the Schrödinger equation cannot be separated, the mixing between 1D subbands is strong and the motion is essentially two-dimensional. Electron transmission can only be considered one-dimensional when the first subband is opening and the transmission coefficient $T<1$.

These assumptions of one-dimensional transmission in device A and two-dimensional transmission in device B are supported by numerical calculations of multiple-mode transmission, as described in the next subsection.

\subsection{Electron transmission through quantum dots}

Two-dimensional transmission was calculated on the same grid in variables $(x, y)$ as was used when the Poisson equation was solved for $U(x, y)$. Along the channel axis $x$, energy levels $E_{n}$ in each transverse cross section and transfer-matrix elements between adjacent cross sections were determined and then the multiple-mode transmission problem was solved by means of scattering $S$-matrices $[25,26]$. The conductance relates to the total transmission coefficient according to the Landauer formula:

$$
G=\frac{2 e^{2}}{h} T \quad T=\sum_{n} T_{n} \quad T_{n}=\sum_{k}\left|T_{n k}\right|^{2} .
$$

The transmission was calculated for quantum dots and single constrictions (half the quantum dot). In figure 7(a) plots of the Fermi energy dependence of the total transmission coefficient and its modal contributions are shown for device A. The dashed lines show the transmission through single constrictions. When the first mode is 50\% transmitted, the second mode has already reached $30 \%$ and so on. For small values of the transverse quantum $\hbar \omega=E_{2}-E_{1}=0.2 \mathrm{meV}$, conductance quantization is smeared out on a single constriction, though it can occur for resonant transmission through two barriers in series. Transmissions through the quantum dot are shown by the solid lines in figure 7. For device A the transmission curves for the first to third subbands resemble each other but with an offset in energy caused by the transverse quantum. Similar behaviour is observed in the split-gate-voltage dependence of the transmission in figure 8 , which models the experimental situation.

It is important that only a few resonant features are present in $T_{n}(E)$ and $T_{n}\left(V_{\mathrm{SG}}\right)$ those are Fabry-Pérot resonances in the system of two barriers. The narrowest resonances are marked with triangles and relate to the tunnelling regime of the corresponding subbands; they are smeared out in measurements and not visible in experiment since in this regime the transport is sequential rather than coherent. In contrast, the wide resonances relate to above-barrier coherent transmission (marked with asterisks) and give rise to every other step of conductance quantization in figures $1-3$. It seems that the conductance steps are not the property of a single barrier, but the property of the pair of barriers (see figures 8 and 7(a)). The difference in height of the barriers of $0.1-0.2 \mathrm{meV}$ (weak asymmetry of the structure) causes no subband mixing but reduces the conductance steps and shifts the resonances (e.g. the dotted curve in figure 7). This asymmetry can explain the observed transformation of the background conductance $\bar{G}_{\mathrm{RA}}\left(V_{\mathrm{SG}}\right)$ in figure 3(a). Firstly, the tops of the barriers in the constrictions approach the Fermi level with a large negative voltage on the finger gates. The inevitable 

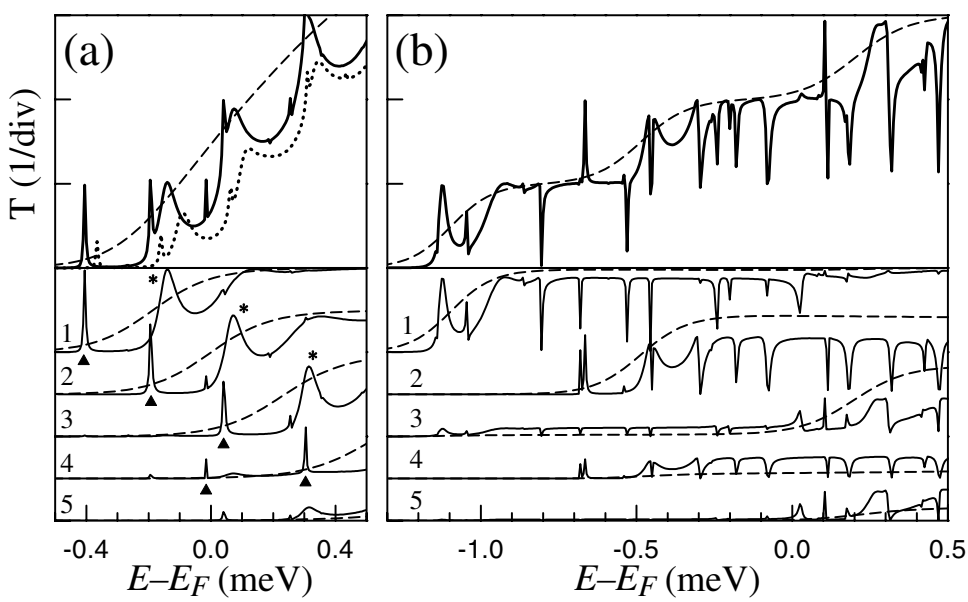

Figure 7. Total transmission coefficient and contributions of 1-5 1D subbands for devices of types $\mathrm{A}\left(V_{\mathrm{SG}}=-0.49 \mathrm{~V}, V_{\mathrm{F}}=-1.4 \mathrm{~V}\right)$ and $\mathrm{B}\left(V_{\mathrm{SG}}=-0.5 \mathrm{~V}, V_{\mathrm{F}}=-1.6 \mathrm{~V}\right)$, in columns (a) and (b) respectively. Dashed lines show corresponding transmission coefficients for a single constriction. The tunnelling resonances are marked with triangles and the above-barrier resonances are marked by asterisks. The dotted line shows the transmission coefficient for device A with the heights of the barriers differing by $0.2 \mathrm{meV}$.

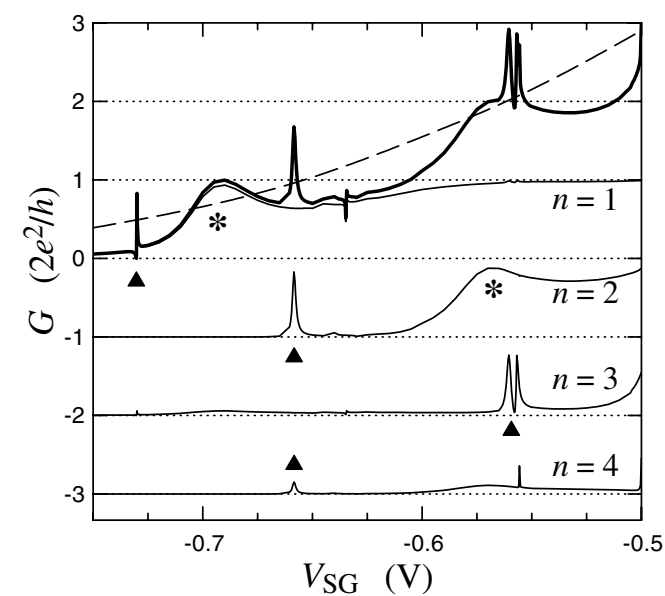

Figure 8. Calculated conductance and contributions of the lowest $1 \mathrm{D}$ subbands as functions of side-gate voltage. The voltages on the finger gates are $V_{\mathrm{F}}=-1.3 \mathrm{~V}$ and $V_{\mathrm{F} 2}=0$. The dashed line shows transmission for a single constriction.

small asymmetry between the two constrictions will be reflected in the transport properties as reduced height of the steps and resonant peaks of the conductance. Secondly, smearing of the features that occurs at lower negative voltages $V_{\mathrm{SG}}$, when $V_{\mathrm{F} 1}$ and $V_{\mathrm{F} 3}$ increase, is favoured by widening of the constrictions in this voltage range, thereby reducing the inter-subband spacing there. And lastly, a decrease of the transparency of the barriers increases the electron dwell time in the dot and the role of decoherence, such that the constrictions start acting independently. The quantized conductance steps for single constrictions smear out in this voltage regime as shown by both measurements (see reference [27], figure 2(a)) and modelling (figures 7 and 8 ). 
The total transmission coefficient and modal distribution for device B are shown in figure 7(b). Dashed curves show transmission with pronounced steps for a single constriction. Because of the large subband spacing, the tunnelling in closed subbands is negligible. In transmission through the dot, however, the transport may go via the higher subbands due to mixing with lower open subbands. For instance, nonzero transmission via the third subband occurs due to coupling to the first subband even if the second subband is not yet transmitted. For $2 e^{2} / h<G<6 e^{2} / h$, the transport involves more than five modes and higher modes contribute much more to the conductance than those in device A. The inter-subband mixing shows up in the $T\left(E_{\mathrm{F}}\right)$ dependence as sharp Fano resonances due to electron scattering from the levels of the dot (figure $7(\mathrm{~b})$ ). The dependence $T\left(V_{\mathrm{SG}}\right)$ is similar to $T\left(E_{\mathrm{F}}\right)$ : transmission is one-dimensional at $T<1$ and already multimodal at $T>1$. It should be noted that while 1D subbands in device A become absolutely transparent $\left(T_{n}=1\right)$ with increasing energy or $V_{\mathrm{SG}}$, the transparency of open subbands in device B changes resonantly from 0 to $80-90 \%$ due to inter-subband mixing. This can explain why charging effects are smeared out at $T>1$ in more standard quantum dots.

Figure 9 shows the modelled dependence of the conductance on the central finger-gate voltage $G\left(V_{\mathrm{F} 2}\right)$. The corresponding deformation of the potential in the dot and constrictions was shown in figures 5(b), 5(c). The depth of the potential in the dot decreases, and the resonances due to $1 \mathrm{D}$ interference on the two barriers cross the Fermi level one by one. The calculated coherent transmission is shown in figure 9 by the solid curve from which the dashed curve without sharp peaks is obtained by smoothing. Five wide Fabry-Pérot resonances are clearly seen in the figure which are also present on the experimental curve (figure 1(a)).

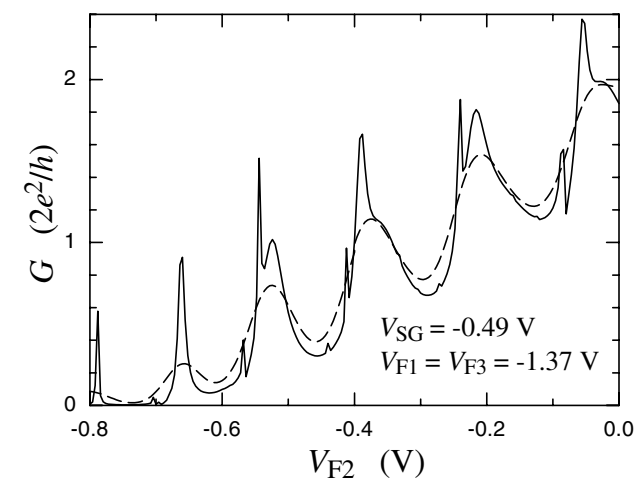

Figure 9. Calculated conductance of the dot as a function of central finger-gate voltage $V_{\mathrm{F} 2}$. The dashed line shows the result of smoothing.

The fact that the number of frequent oscillations in experimental curves differs drastically from the number of resonant features in the calculated transmission coefficients (figures 8 and 9) demonstrates that the observed frequent oscillations are not due to interference effects of coherent electron transmission through quasi-discrete states of the quantum dot. In the coherent regime an electron does not scatter on most levels in the absence of mode mixing. The suppression of mode mixing is a consequence of the geometry of the dot and the corresponding selected range of voltages at finger and side gates. Large negative voltage at overlying finger gates flattens the potential across the channel with the result that the separation between the lowest 1D subbands in the constrictions becomes as small as that in the dot (figure 6(e)). Calculations of the transmission coefficients show that mode mixing is strengthened when the conductance rises to $G \approx 6 e^{2} / h$. 


\subsection{The mechanism of single-electron charging of open quantum dots}

On the basis of the modelling of the electrostatics and coherent transmission, we suggest the following scenario of Coulomb charging in an open quantum dot. There are three important features that the new type of dot possesses: (1) coupling between 1D subbands is suppressed; (2) coherent transmission and sequential tunnelling coexist; (3) the charging energy and 1D subband spacing in the constrictions are commensurate.

Suppression of inter-subband mixing implies the presence of localized states of closed subbands against the background of current-carrying states. Weak sequential tunnelling causes the recharging of localized states and leads to a modulation of the potential of the dot via Coulomb blockade. Taking this modulation into account by means of the Landauer formula will produce single-electron oscillations in the coherent ballistic current. The problem is to relate the population of the delocalized states to the charge of the localized states. Below, we describe how the modulation of the potential is calculated, also giving an account of this relation, and suggest a modification of orthodox theory of Coulomb blockade (OTCB) for the open quantum dot with suppressed 1D subband mixing. From the response of the transmission coefficient to the change of the potential we obtain, with the help of the modified theory, the gate-voltage dependence of the conductance containing single-electron oscillations.

If the $1 \mathrm{D}$ subbands in the quantum dot do not mix, then there are many localized states near the Fermi level, with the background of 1-3 delocalized states (figure 10). In this case the resonance peaks of the transmission coefficient only correspond to the open 1D subbands (Fabry-Pérot resonances, figure 7(a)) while the localized states are not displayed since they are too narrow. The decay of localized states via the open subbands has a low probability, so the quantum fluctuations of charge in the dot are suppressed. The states of open and closed $1 \mathrm{D}$ subbands can be considered as two systems separated by effectively thick barriers. Due to these barriers, the system of localized states of the open dot remains under the conditions where the OTCB is applicable. That is, the localized states can capture an electron or return it to the reservoir by means of sequential tunnelling through the effectively thick potential barriers. Between the tunnelling events the system relaxes to one of several states of electrostatic equilibrium. Each of these states is entirely described by the voltage difference $V_{\mathrm{b}}$ between the dot and the reservoir with the 2DEG. In the limit of zero voltage between source and drain

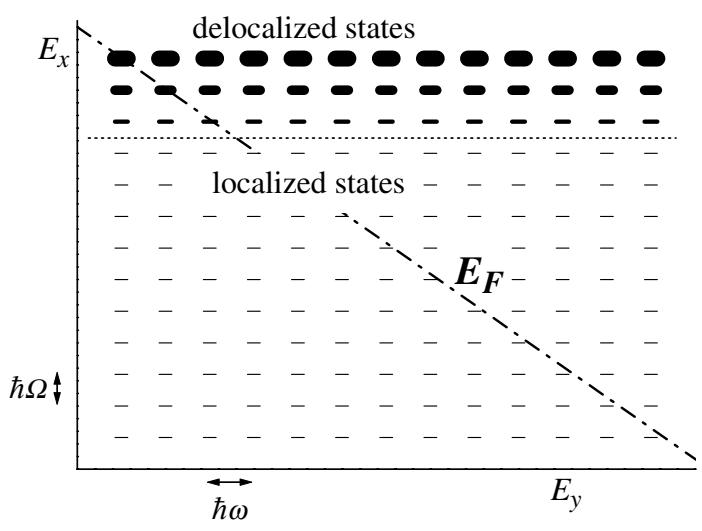

Figure 10. A schematic drawing of single-particle levels of an open quantum dot with suppressed inter-subband mixing. $E_{x}$ and $E_{y}$ denote the kinetic energy for motion along and across the dot, respectively. The columns correspond to $1 \mathrm{D}$ subbands. The boundary between localized and delocalized states is marked by the dotted line. 
reservoirs, the value $V_{\mathrm{b}}$ is determined from a simple electrostatic relation:

$$
C V_{\mathrm{b}}=n e+C_{\mathrm{g}} V_{\mathrm{g}}+q_{0}
$$

where $C=C_{1}+C_{2}+C_{\mathrm{g}}, C_{1}$ and $C_{2}$ are the reservoir-dot capacitances, $n$ the number of electrons in the localized states and $q_{0}$ is a free parameter in the OTCB, called the polarization charge of the Coulomb island. The value of $q_{0}$, like that of $C_{\mathrm{g}} V_{\mathrm{g}}$, need not be an integer in units of electron charge $e$. In the OTCB the polarization charge is assumed constant and describes the interaction of the isolated dot (Coulomb island) with uncontrollable charges and dipoles.

In the limit of zero temperature, constant $q_{0}$ would lead to a sharp step-like dependence $\bar{n}\left(C_{\mathrm{g}} V_{\mathrm{g}}\right)$, where the overline implies averaging over the states of electrostatic equilibrium, and hence a sawtooth modulation of $\overline{V_{\mathrm{b}}}$ between $-e / 2 C$ and $e / 2 C$ as the value $C_{\mathrm{g}} V_{\mathrm{g}}$ changes by $e$. The system under study differs fundamentally from an isolated dot since $q_{0}$ is not a free parameter but depends self-consistently on the charge of the localized states $n e$. This requires the theory of $\mathrm{CB}$ to be modified. In our case the role of polarization charge is played by the charge that belongs to open subbands. This charge is defined by the population of transmitted, or open 1D subbands (the $i$ th subband is transmitted if its bottom $E_{i}$ remains in the narrowest part $x_{\mathrm{c}}$ of the constriction below the Fermi level, $E_{i}\left(x_{\mathrm{c}}\right)=U\left(x_{\mathrm{c}}\right)+\hbar \omega\left(i+\frac{1}{2}\right)$, where $U\left(x_{\mathrm{c}}\right)$ is the top of electrostatic barrier; see figure 6(e)). The subbands are shifted when the voltage drop between the dot and reservoirs $V_{\mathrm{b}}$ changes in response to recharging of localized states by tunnelling or varied gate voltage.

We define the polarization charge as $q_{0}=q_{00}+\sum q_{0 i}$, where $q_{00}$ is constant and the contribution $q_{0 i}$ of the $i$ th open subband is expressed in terms of the density of delocalized states in $1 \mathrm{D}$ subbands $(\hbar \Omega)^{-1}$ and subband occupation $\Delta E_{i}=E_{\mathrm{F}}-U\left(x_{\mathrm{c}}\right)-\hbar \omega\left(i+\frac{1}{2}\right)$ as

$$
q_{0 i}= \begin{cases}2 e \Delta E_{i} / \hbar \Omega & \Delta E_{i}>0 \\ 0 & \Delta E_{i} \leqslant 0 .\end{cases}
$$

Here we use the approximation of a parabolic quantum dot with

$$
U(x, y) \approx U(0,0)+\frac{1}{2} m^{*}\left(\omega^{2} y^{2}+\Omega^{2} x^{2}\right) .
$$

To complete the set of equations we shall relate $\Delta E_{i}$ to $V_{\mathrm{b}}$ and $V_{\mathrm{g}}$. We assume that the height of the barrier in the constriction $U\left(x_{\mathrm{c}}\right)$ is shifted by $\frac{1}{2} e V_{\mathrm{b}}$ under the voltage drop $V_{\mathrm{b}}$ over the barrier, $U\left(x_{\mathrm{c}}\right)=\left.U\left(x_{\mathrm{c}}\right)\right|_{V_{\mathrm{b}}=0}+\frac{1}{2} e V_{\mathrm{b}}$.

We define the dependence $\Delta E_{i}\left(V_{\mathrm{g}}\right)$ phenomenologically under the assumption that $V_{\mathrm{g}} \equiv V_{\mathrm{SG}}$ and subbands open sequentially with equal steps $\delta V_{\mathrm{g}}$ starting from $V_{g 0}$; thus $\Delta E_{i}=$ $\hbar \omega\left[\left(V_{\mathrm{g}}-V_{\mathrm{g} 0}\right) / \delta V_{\mathrm{g}}-i\right]$. From modelling the electrostatics (figure 4 , insets), $\hbar \omega$ is known to decrease by a factor of 2 as $V_{\mathrm{g}}$ changes by $2 \delta V_{\mathrm{g}}$; that is, $\hbar \omega=\hbar \omega_{0}\left[\frac{1}{2}\left(V_{\mathrm{g}}-V_{\mathrm{g} 0}\right) / \delta V_{\mathrm{g}}+1\right]^{-1}$, where $\hbar \omega_{0}$ corresponds to opening of the first subband. Therefore we eventually obtain

$$
\Delta E_{i}=-\frac{e V_{\mathrm{b}}}{2}+\hbar \omega_{0} \frac{\left(V_{\mathrm{g}}-V_{\mathrm{g} 0}\right) / \delta V_{\mathrm{g}}-i}{\frac{1}{2}\left(V_{\mathrm{g}}-V_{\mathrm{g} 0}\right) / \delta V_{\mathrm{g}}+1} .
$$

Taking account of the thermal washing, the expression for $q_{0}$ can now be written as

$$
q_{0}=q_{00}+\frac{2 e}{\hbar \Omega} \sum_{i} \frac{\Delta E_{i}}{1-\exp \left(-\Delta E_{i} / k_{\mathrm{B}} T\right)} .
$$

Thus unlike in the OTCB, $q_{0}$ is a function of $V_{\mathrm{b}}$ and $V_{\mathrm{g}}$ in our case, and the calculations with equations (1)-(4) will give different results.

Figure 11(a) shows the calculation of the dot-reservoir voltage $\overline{V_{\mathrm{b}}}\left(V_{\mathrm{g}}\right)$ averaged over possible charge states. One can see that single-electron oscillations are present at $T=50 \mathrm{mK}$ in the whole voltage range from $-680 \mathrm{mV}$ to $-480 \mathrm{mV}$. The amplitude of the oscillations 


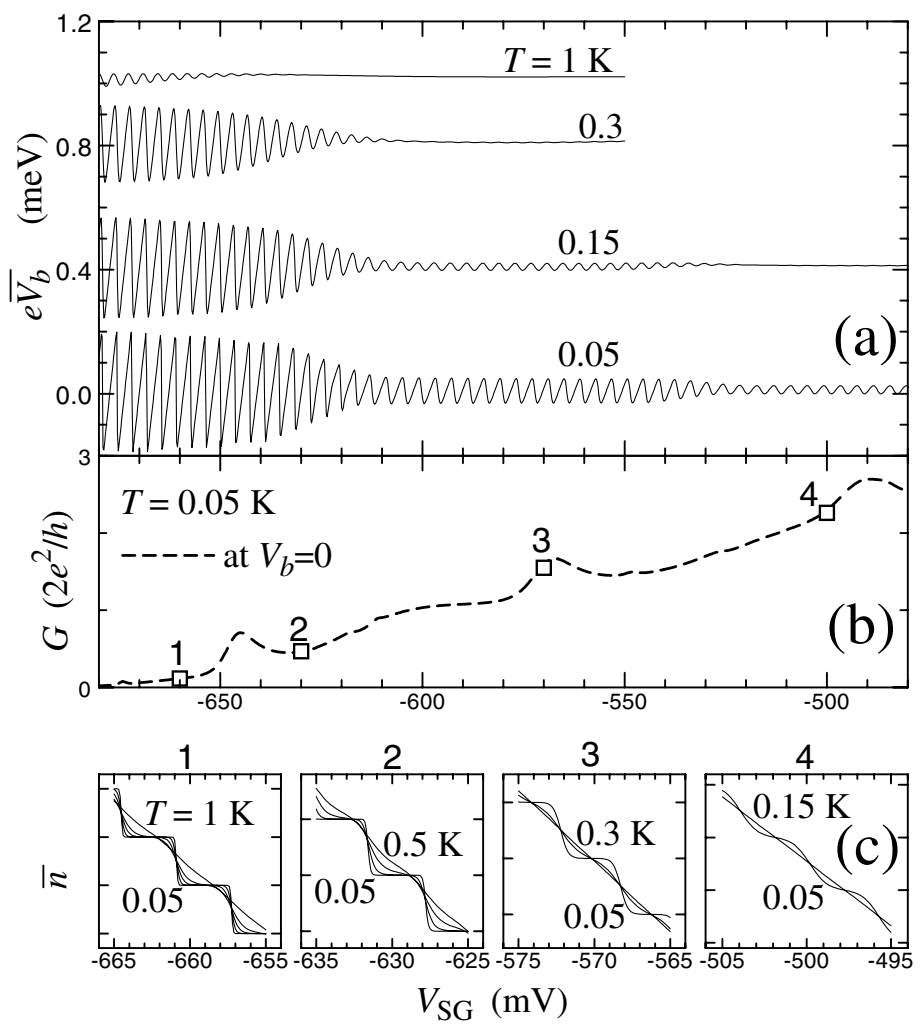

Figure 11. Modelling single-electron charging of quantum dots with suppressed inter-subband mixing. (a) The sawtooth dependence of the voltage drop $e \overline{V_{\mathrm{b}}}$ over inlet/outlet barriers on split-gate voltage $V_{\mathrm{SG}}$ at different temperatures. (b) An illustration of subband filling: calculated conductance of the dot with $V_{\mathrm{b}}=0$. (c) Fragments of step-like dependences of the number of electrons in the localized states of the quantum dot for relevant temperatures from the set $\{0.05,0.15,0.3$, 0.5 and $1 \mathrm{~K}$ \}. The enumeration in (c) is related to the conductance in (b): 1 - Coulomb blockade, 2-transition to the open dot, 3-the first subband is fully transmitted, 4-the second subband is fully transmitted.

corresponds to the charging energy $E_{Q}\left(V_{\mathrm{g}}\right)$ of the localized states with account taken of the population of open 1D subbands. On the left of the voltage range the dot is closed and the amplitude of the oscillations reaches the value $e / 2 C$, the same as in Coulomb blockade theory. To the right the amplitude decreases by more than an order of magnitude when the second 1D subband is fully open. Actually this implies a respective decrease of the critical temperature at which the charging effects are still observed, according to the rule $E_{Q} \sim k_{\mathrm{B}} T_{\mathrm{c}}$. The amplitude of oscillations decreases in a step-like fashion as the 1D subbands successively open, each giving a contribution of $e^{2} / \hbar \Omega$ to the effective capacitance of the dot. To illustrate the filling of $1 \mathrm{D}$ subbands, figure 11(b) shows the conductance calculated using equation (5) with $V_{\mathrm{b}}=0$, as will be discussed later. To show how the quantization of charge in the localized states of the quantum dot is smeared out with increasing temperature, we provide the calculated $\bar{n}$ (figure 11(c)) and $\overline{V_{\mathrm{b}}}$ (figure 11(a)). For low temperature, the suggested model describes a transition from Coulomb blockade to suppressed quantization of charge of the localized states in open quantum dots, and this transition extends over the voltage range where the three lowest 1D subbands become successively occupied. The calculation also shows that the oscillations 
in $\overline{V_{\mathrm{b}}}$ and steps in $\bar{n}$ completely disappear at $T \approx 0.3 \mathrm{~K}$ as soon as the first subband becomes transmitted (figures $11(\mathrm{a}), 11(\mathrm{c}))$. At $T=1 \mathrm{~K}$ the interval with oscillations shrinks, and no oscillations are observed for $T=2 \mathrm{~K}$ which is the critical temperature for the isolated quantum dot as discussed in reference [23].

\subsection{Coulomb oscillations of ballistic conductance}

On the basis of our model of charging, we have tested that if the effective barrier resistance $R>R_{Q}=h / e^{2}$, the current of the sequential tunnelling would produce oscillations much weaker than those measured experimentally. Hence the observed oscillations are determined not only by the current through the localized states, but also by the ballistic current. In section 3.2 we discussed the feature that the spacing of $1 \mathrm{D}$ subbands $\hbar \omega$ in the constrictions is approximately equal to the charging energy $e^{2} / 2 C=0.1-0.2 \mathrm{meV}$, so we expected high sensitivity of the coherent ballistic conductance to the variations of $V_{\mathrm{b}}$. We have numerically estimated that the transparency of the quantum dot changes by $0.3\left(e^{2} / h\right)$ when $V_{\mathrm{b}}$ is varied by only $0.1 e / C$ (the bottom of the dot is raised by $0.1 e^{2} / C$ ). Thus, the periodic change of the embedded voltage $V_{\mathrm{b}}$ with gate voltage will result in single-electron conductance oscillations in the coherent current. Therefore we calculated the ballistic conductance in the presence of single-electron modulation of the electrostatic potential by employing an extended Landauer formula which takes into account the probability distribution $P_{n}$ of charge states:

$$
G=\frac{2 e^{2}}{h} \sum_{n} P_{n} \int \sum_{i} T_{i}\left(E, V_{\mathrm{b} n}, V_{\mathrm{g}}\right) F\left(E-E_{\mathrm{F}}\right) \mathrm{d} E
$$

where $F(E)=\left(1 / 4 k_{\mathrm{B}} T\right) \operatorname{sech}^{2}\left(E / 2 k_{\mathrm{B}} T\right)$. We simplified the calculations by assuming that $T_{i}=T_{0}^{*}\left(E-\Delta E_{i}\left(V_{\mathrm{b} n}, V_{\mathrm{g}}\right)\right)$, where $T_{0}^{*}(E)$ is the transmission coefficient in the first subband shown in figure 7(a). Reducing $T_{i}$ to $T_{0}^{*}$ is plausible with absence of inter-subband mixing assumed. In reality, the phase breaking of the wave function that does not lead to capture of ballistic electrons by localized states makes $T_{0}^{*}$ take an intermediate value between the coefficients of coherent transmission through a double barrier $T_{2 \mathrm{~b}}$ and sequential transmission through two barriers in series $\frac{1}{2} T_{1 \mathrm{~b}}$. The phase-breaking events become rarer with increase of transparency of the single barrier $T_{1 \mathrm{~b}}$, so we used $T_{0}^{*}=\alpha T_{2 \mathrm{~b}}+\frac{1}{2}(1-\alpha) T_{1 \mathrm{~b}}$ with weight coefficient $\alpha=T_{1 \mathrm{~b}}$.

Figure 12(a) shows the results of conductance calculations carried out using the modified (equation (5)) and usual ( $V_{\mathrm{b}}=0$ and $T_{i}$ independent of $n$ ) Landauer formulae. For low temperatures the conductance $G\left(V_{\mathrm{g}}\right)$ calculated with no account taken of single-electron charging is similar to that calculated in section 3.3 (figure 8) except for narrow features which are smeared out here due to $\alpha$. This similarity of the curves justifies reducing $T_{i}$ to $T_{0}^{*}$. The accounting for single-electron modulation of the potential results in single-electron oscillations of ballistic conductance. At low temperatures the suggested model describes the oscillations which are superimposed on broad Fabry-Pérot resonances and cover the range $0<G<6 e^{2} / h$. The modulation of their amplitude is caused by increased sensitivity of the conductance to the variations of $V_{\mathrm{b}}$ on the slopes of large-scale peaks. When the temperature increases to $T=0.15 \mathrm{~K}$ the oscillations at $G>4 e^{2} / h$ are suppressed. At $T=0.3 \mathrm{~K}$ the oscillations are already suppressed above $2 e^{2} / h$ and interference peaks are smoothed. At $T=1 \mathrm{~K}$ the oscillations survive only for $G<e^{2} / h$. Rapid disappearance of large-scale peaks cannot be explained by just thermal broadening of the energy of incident particles; another reason is single-electron modulation of the potential. With increasing temperature the number of states participating in charging of the dot grows and the transmission coefficient as a function of gate voltage is strongly averaged and smoothed. For comparison, figure 12(b) shows 

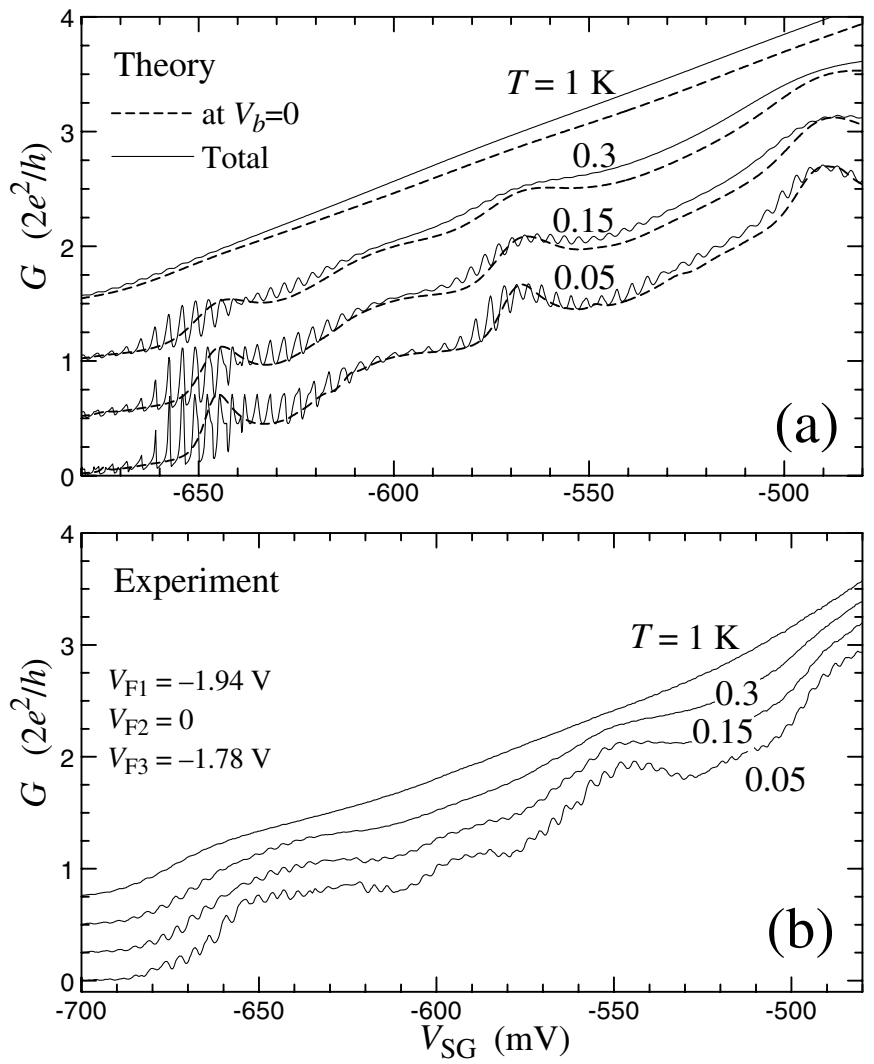

Figure 12. Calculated (a) and measured (b) conductance of the quantum dot as a function of splitgate voltage. Dashed lines show conventional Landauer conductance, with no account taken of the single-electron oscillations in $V_{\mathrm{b}}\left(V_{\mathrm{b}}=0\right)$. Solid curves demonstrate single-electron oscillations of the conductance around Fabry-Pérot resonances, as obtained with (5) which involves dependence of $V_{\mathrm{b}}$ on $V_{\mathrm{SG}}$ and a probability distribution of charge states of the dot.

measured dependences $G\left(V_{\mathrm{g}}\right)$ for the same temperatures. One can see that the calculations are in good agreement, reproducing all relevant features of the background and oscillations. Notice that the period of single-electron oscillations in calculations fluctuates, $\Delta V_{\mathrm{SG}}=3.8 \pm 1 \mathrm{mV}$, while in experiment it is $\Delta V_{\mathrm{SG}}=3.6 \pm 1 \mathrm{mV}$. The main difference from the experimental plot is that the calculated curves have greater amplitude and are more pronounced, which is a consequence of the simplified expression for $q_{0}$ and the overestimated contribution of the resonant transmission near the threshold. Despite such simplifications, the model correctly reproduces critical temperatures for observing the oscillations.

We tried to model the charging of standard split-gated open quantum dots with strong inter-subband scattering. In this case (figure 7(b)) there are many Fano resonances in $T_{\text {tot }}(E)$ and the number of quickly decayed states in open subbands is much greater than the number of Fabry-Pérot resonances in the 1D system studied. Additionally, because of strong intersubband mixing, the third $(i=2)$ subband becomes partially transparent simultaneously with the opening of the first $(i=0)$ subband. These two factors make the density of states $(\hbar \Omega)^{-1}$ in more standard quantum dots redistribute in favour of open subbands, in comparison with the 1D case. In our model we incorporate this redistribution by increasing the effective capacitance $e^{2} / \hbar \Omega$ of each open subband. We have found that decreasing $\hbar \Omega$ by a factor of 2 makes 
the low-temperature oscillations of the conductance disappear as soon as the second subband ( $i=1$ ) begins to open. On reducing $\hbar \Omega$ to a third of the original value, the conductance oscillations disappear as soon as the lowest subband $(i=0)$ opens. Hence, although the model is developed for 1D systems and does not use quantum fluctuations of charge, it shows that electrostatic interaction of charge in delocalized and localized states would suffice to suppress Coulomb oscillations of conductance propagating into the region $G>2 e^{2} / h$ for more standard quantum dots of the same size. Moreover, this calculation shows that increasing the length of the dot would suppress single-electron oscillations at $G>2 e^{2} / h$ as well. In a small quantum dot, on the other hand, Fabry-Pérot resonances are feebly marked, with large spacing, so the ballistic conductance will sense single-electron modulation of the potential and produce the related oscillations only on the slopes of the quantization steps, between the conductance plateaux. This was the case, we suppose, when such oscillations were observed up to $G=14 e^{2} / h$ in ballistic conductance of a small dot formed by an impurity potential [20]. Thus, the model that we suggested allows us to describe different extents of progression of Coulomb oscillations into the quantum ballistic conductance depending on temperature, size and dimensionality of the quantum dots.

\section{Conclusions}

In this paper we have confirmed the single-electron nature of conductance oscillations of open quantum dots reported in reference [23], and clarified the mechanism of the effect. In experiment the oscillations are proof against perpendicular magnetic field, as they should be for single-electron charging. By means of calculations of capacitances of the dot to the gates and 2DEG, we have found that the period of the oscillations corresponds to addition of a single electron to the dot and the charging energy agrees with the measured temperature of disappearance of conductance oscillations at $G<e^{2} / h$. 3D calculations of the electrostatic potential of the device, combined with solution of the $2 \mathrm{D}$ scattering problem, show that the transport through this dot is almost one-dimensional, in contrast to the case for standard split-gated quantum dots with strong inter-subband scattering. That the system studied is onedimensional means that inter-subband mixing is suppressed and the number of quasi-discrete states in transmitted subbands (Fabry-Pérot resonances) is small. The larger part of the charge of the dot belongs to the localized states of closed subbands and only changes in rare events of sequential tunnelling. Quantum fluctuations of this charge are suppressed together with intersubband mixing. Since the number of delocalized states is small, the contribution of transmitted subbands to charging of the dot is limited, and the charge of the localized states of closed 1D subbands remains quantized at low temperatures. As a result, charging of the localized states by a weak tunnelling current leads to single-electron modulation of the electrostatic potential of the dot as the gate voltage is varied. According to the Landauer formula this modulation gives rise to single-electron oscillations in ballistic conductance, added to the background of quantization steps and interference resonances. It should be noted that in this 1D system there is a weakly transparent effective barrier which separates the localized states of closed subbands from the reservoirs. That is why it is difficult to observe the sharp equidistant peaks of sequential tunnelling simultaneously with strong and smoothed oscillations of ballistic current. Thus, we believe that in the system studied the effect of charge quantization is manifested in the ballistic current. To model this effect quantitatively, we modified the conventional theory of Coulomb blockade and the Landauer formula, and correctly reproduced single-electron and interference features of ballistic conductance, including their smoothing and disappearance with temperature. 


\section{Acknowledgments}

This work was supported by programmes of the Ministry of Science of the Russian Federation: 'Physics of Solid-State Nanostructures' (98-1102) and 'Prospective Technologies and Devices for Micro- and Nanoelectronics' (02.04.5.1), and by the programme 'Universities of RussiaFundamental Research' (1994). The work at Cambridge was funded by the Engineering and Physical Sciences Research Council (EPSRC), UK. CTL is grateful for financial support from the NSC (90-2112-M-002-033). We are grateful to Kvon Ze Don, M V Éntin, C H W Barnes and V I Talyanskii for discussions. OAT and DGB thank colleagues from the Cavendish Laboratory, especially C J B Ford, for fruitful discussions and hospitality.

\section{References}

[1] Likharev K K 1988 IBM J. Res. Dev. 32144

[2] Glazman L I and Shekhter R I 1989 J. Phys.: Condens. Matter 15811

[3] Smith C G et al 1988 J. Phys. C: Solid State Phys. 21 L893

[4] Meirav U, Kastner M A and Wind S J 1990 Phys. Rev. Lett. 65771

[5] McEuen P L et al 1991 Phys. Rev. Lett. 661926

[6] Weis J et al 1993 Phys. Rev. Lett. 714019

[7] Ashoori R C et al 1992 Phys. Rev. Lett. 683088

[8] Staring A A M et al 1991 Physica B 175226

[9] Kouwenhoven L P et al 1991 Z. Phys. B 85367

[10] Williamson J G et al 1992 Nanostructures and Mesoscopic Systems ed W P Kirk and M A Reed (New York: Academic) p 255

[11] Foxman E B et al 1993 Phys. Rev. B 4710020

[12] Heinzel T et al 1994 Europhys. Lett. 26689

[13] Persson M et al 1995 Phys. Rev. B 528921

[14] Matveev K A 1995 Phys. Rev. B 511743

[15] Nazarov Y V 1999 Phys. Rev. Lett. 821245

[16] Aleiner I L and Glazman L I 1998 Phys. Rev. B 579608

[17] Glazman L I, Hekking F W J and Larkin A I 1999 Phys. Rev. Lett. 831830

[18] Pasquier C et al 1993 Phys. Rev. Lett. 7069

[19] Field M et al 1994 J. Phys.: Condens. Matter 6 L273

[20] Liang C-T et al 1997 Phys. Rev. B 556723

[21] Möller C et al 1998 Phys. Rev. Lett. 815197

[22] Cronenwett S M et al 1998 Phys. Rev. Lett. 815904

[23] Liang C-T et al 1998 Phys. Rev. Lett. 813507

[24] Zailer I et al 1996 Semicond. Sci. Technol. 111235

[25] Tkachenko O A et al 2000 Pis. Zh. Eksp. Teor. Fiz. 71366 (Engl. Transl. JETP Lett. 71 255)

[26] Tkachenko O A et al 2001 J. Appl. Phys. 894993

[27] Liang C-T et al 1999 Phys. Rev. B 6010687 